Version: ANU, November 13, 2018

\title{
Quantum field effects in coupled atomic and molecular Bose-Einstein condensates
}

\author{
J. J. Hope \\ Department of Physics and Theoretical Physics, Australian National University, \\ ACT 0200, Australia.
}

\begin{abstract}
This paper examines the parameter regimes in which coupled atomic and molecular Bose-Einstein condensates do not obey the Gross-Pitaevskii equation. Stochastic field equations for coupled atomic and molecular condensates are derived using the functional positive-P representation. These equations describe the full quantum state of the coupled condensates and include the commonly used Gross-Pitaevskii equation as the noiseless limit. The model includes all interactions between the particles, background gas losses, twobody losses and the numerical simulations are performed in three dimensions. It is found that it is possible to differentiate the quantum and semiclassical behaviour when the particle density is sufficiently low and the coupling is sufficiently strong.
\end{abstract}

PACS numbers: 03.75.Fi,03.75-b,32.80.Wr 


\section{INTRODUCTION}

One of the surprising discoveries that has been made since the experimental production of a Bose-Einstein condensate (BEC) in a weakly interacting gas [四] has been the fact that virtually all of their properties can be described using the Gross-Pitaevskii equation (GPE). This semiclassical approximation ignores any dynamics in the quantum statistics of the field. There has been much interest recently in the production of a molecular Bose-Einstein condensate (MBEC) from the photoassociation of an atomic BEC of a weakly interacting dilute gas [2 13]. We have recently calculated that in certain parameter regimes the GPE can give incorrect results for this process [14]. This paper investigates the process with a three-dimensional model that includes background gas losses, two-body losses and the atom-molecule interactions.

The success of the GPE [15] might not seem so surprising considering the effectiveness of the semiclassical approximation in quantum optics [17]. It includes the effects of s-wave interactions, and can be readily generalised to include multi-component condensates with inter-species couplings [4, 16]. As a semiclassical, mean-field theory it necessarily cannot give information about the quantum statistics of the condensates, but for most experiments with BEC these properties have not been observed. Quantum statistics affect some nonlinear quantum optical systems, the simplest of which is second harmonic generation, where pairs of photons are coupled to single, high-energy photons [18. The analogous process in atom optics is that of the coupling of a MBEC and a BEC, which may be done either through tuning of a Feshbach resonance [11], or through photoassociation via a two-photon Raman coupling [4].

The Bose-enhancement of the photoassociation of atoms from a trapped BEC leads to giant, collective oscillations between the atomic and molecular populations. This enhancement of a chemical process was dubbed "superchemistry" by Heinzen et al. when they first modeled it using a two-component Gross-Pitaevskii equation (GPE) [4. A more recent model using the Hartree-Fock-Bogoliubov method includes pair correlations in the atomic field, and showed discrepancies with the results obtained with the GPE [11]. The full effects of the quantum nature of the fields can be modeled by a set of stochastic equations for the atomic and molecular fields based on the functional positive-P representation [19 22]. A recent paper used this technique in a one-dimensional calculation to show that it was possible to see the effects of the quantum statistics in the population dynamics of coupled atomic and molecular BEC [14]. The signature was a reduction in the transfer from atoms to molecules. This paper uses a more complete model which includes all three dimensions as well as atom-molecule interactions and losses. The parameter regimes in which the GPE breaks down are determined.

In Sec. II the extended model is described and the stochastic equations of motion for the system are derived. The following section is a brief examination of the behaviour of the GPE and the likely parameter regimes in which there are measurable deviations from this behaviour. In Sec. IV the evolution of the atomic population is compared with the solution of the GPE for a range of densities and coupling strengths. 


\section{MODEL}

An atomic field is coupled to a molecular field by two-colour Raman photoassociation. A first laser couples a single electronic level of the atomic field to a set of molecular excited states. A second laser then couples these states to a stable molecular level. The modes are arranged as shown in Fig. 1, with state $|1\rangle$ being the atomic BEC, state $|2, \nu\rangle$ the $\nu$ th vibrational level of the excited state of the MBEC and state $|3\rangle$ the stable MBEC. Two laser fields induce a free-bound coupling between $|1\rangle$ and $|2, \nu\rangle$ and a bound-bound coupling between $|2, \nu\rangle$ and $|3\rangle$. In a rotating frame, the Hamiltonian may be written as

$$
\begin{aligned}
\hat{H} & =\sum_{i=1}^{3}\left(\hat{T}_{i}+\hat{V}_{i}\right)+\sum_{i j} \int d^{3} x \hat{\psi}_{i}^{\dagger}(x) \hat{\psi}_{j}^{\dagger}(x) \frac{U_{i j}}{2} \hat{\psi}_{i}(x) \hat{\psi}_{j}(x) \\
& +\frac{i \hbar}{2} \sum_{\nu} \int d^{3} x\left(\kappa_{\nu}(x) \hat{\psi}_{1}^{\dagger}(x) \hat{\psi}_{2, \nu}(x)-\kappa_{\nu}^{*}(x) \hat{\psi}_{1}^{2}(x) \hat{\psi}_{2, \nu}^{\dagger}(x)\right) \\
& +i \hbar \sum_{\nu} \int d^{3} x\left(\Omega_{\nu}(x) \hat{\psi}_{2, \nu}^{\dagger}(x) \hat{\psi}_{3}(x)-\Omega_{\nu}^{*}(x) \hat{\psi}_{2, \nu}(x) \hat{\psi}_{3}^{\dagger}(x)\right)
\end{aligned}
$$

where $\hat{\psi}_{j}(x)$ is the field annihilation operator for the atomic or molecular field in state $|j\rangle$, $\hat{T}_{i}$ and $\hat{V}_{i}$ are the kinetic and potential energy operators for the $i^{\text {th }}$ field, $U_{i j}$ is the strength of the interatomic interactions between particles in states $|i\rangle$ and $|j\rangle, \kappa_{\nu}(x)$ is the Rabi frequency of the free-bound photoassociation from level $|1\rangle$ to level $|2, \nu\rangle$ and $\Omega(x)$ is the Rabi frequency of the bound-bound transition from level $|2, \nu\rangle$ to level $|3\rangle$. In this notation, the detunings of the lasers from the bare atomic and molecular energy levels are included in the potential energy terms $V_{2, \nu}$ and $V_{3}$. 


\section{FIGURES}

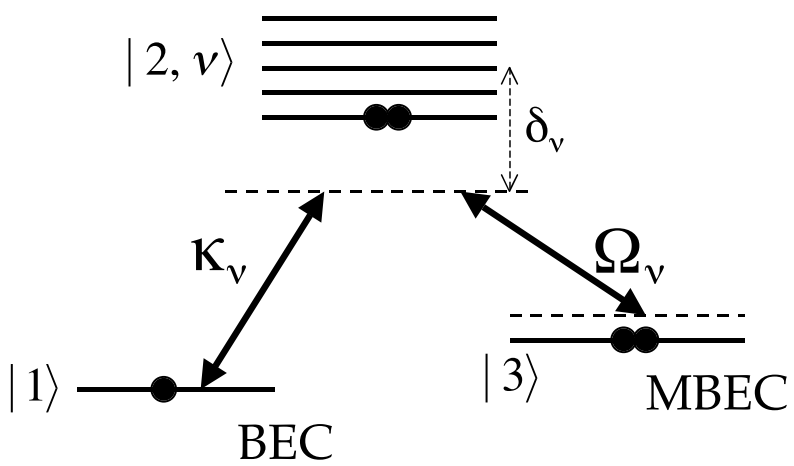

FIG. 1. Energy level scheme for coherent free-bound-bound photoassociation. Levels $|1\rangle,|2, \nu\rangle$ and $|3\rangle$ are the electronic states for the atomic BEC, the $\nu$-vibrational level excited MBEC and the stable MBEC respectively.

In addition to the coherent effects produced by this Hamiltonian, the losses from the trapped levels are included by adding standard loss terms to the master equation. The total master equation is:

$$
\begin{aligned}
\dot{\rho}= & -\frac{i}{\hbar}[\hat{H}, \rho]+\sum_{j} \gamma_{j}^{(1)} \int d^{3} x \mathcal{D}\left[\hat{\psi}_{j}(x)\right] \rho \\
& +\sum_{j} \gamma_{j}^{(2)} \int d^{3} x \mathcal{D}\left[\hat{\psi}_{j}^{2}(x)\right] \rho \\
& +2 \gamma_{13}^{(2)} \int d^{3} x \mathcal{D}\left[\hat{\psi}_{1}(x) \hat{\psi}_{3}(x)\right] \rho
\end{aligned}
$$

where $\gamma_{j}^{(1)}$ is the loss rate from level $|j\rangle$ due to background gases, $\gamma_{j}^{(2)}$ is the two-body loss rate from level $|j\rangle, \gamma_{13}^{(2)}$ is the two-body loss rate due to inelastic collisions between the BEC and MBEC fields, and where the superoperator $\mathcal{D}$ is defined by

$$
\begin{aligned}
\mathcal{D}[c] & =\mathcal{J}[c]-\mathcal{A}[c], \\
\mathcal{J}[c] \rho & =c \rho c^{\dagger}, \\
\mathcal{A}[c] \rho & =\frac{1}{2}\left(c^{\dagger} c \rho+\rho c^{\dagger} c\right) .
\end{aligned}
$$

\section{A. Derivation of the stochastic field equations}

The master equation is a field operator equation with a non-trivial level of excitation, and is therefore impossible to solve numerically by direct means. Analytical results are precluded by the nonlinearities in the equations. To find a numerical method which is tractable in some parameter regimes, this master equation is written in the functional positive- $P$ representation [20,23]

$$
P\left(\left\{\psi^{\alpha}, \psi^{\beta}\right\}, \tau\right)=\left.\rho^{(a)}\left(\left\{\hat{\psi}, \hat{\psi}^{\dagger}\right\}, \tau\right)\right|_{\hat{\psi} \leftrightarrow \psi^{\alpha}, \hat{\psi}^{\dagger} \leftrightarrow \psi^{\beta}}
$$


where $\rho^{(a)}$ is the density operator antinormally ordered with respect to the field operators in the Schrödinger picture. It is then possible to use the functional operator correspondences:

$$
\begin{aligned}
\hat{\psi} \rho \leftrightarrow \psi^{\alpha} P, & \hat{\psi}^{\dagger} \rho \leftrightarrow\left(\psi^{\beta}-\frac{\partial}{\partial \psi^{\alpha}}\right) P, \\
\rho \hat{\psi}^{\dagger} \leftrightarrow \psi^{\beta} P, & \rho \hat{\psi} \leftrightarrow\left(\psi^{\alpha}-\frac{\partial}{\partial \psi^{\beta}}\right) P,
\end{aligned}
$$

to write a functional Fokker-Planck equation (FPE) from the master equation. This FPE may be written in the form:

$$
\frac{\partial P}{\partial t}=\sum_{\nu} \int \mathrm{dx}\left[-\partial_{\nu} A^{\nu}+\sum_{\mu} \frac{1}{2} \partial_{\mu} \partial_{\nu} D^{\mu \nu}\right] P
$$

where the elements $\mu$ and $\nu$ correspond to the $(4+2 n)$ components of the fields in the positive-P representation: $\left\{\psi_{1}^{\alpha}, \psi_{1}^{\beta}, \ldots, \psi_{2, \nu}^{\alpha}, \psi_{2, \nu}^{\beta}, \ldots, \psi_{3}^{\alpha}, \psi_{3}^{\beta}\right\}, A$ is the drift vector, $D$ is the diffusion matrix, and $\partial_{(\nu \leftrightarrow\{n, \gamma\})} \equiv \frac{\partial}{\partial \psi_{n}^{\gamma}}$. The drift vector is given by

$$
A=\left(\begin{array}{c}
\left(\mathcal{K}_{1}-\frac{\gamma_{1}^{(1)}}{2}\right) \psi_{1}^{\alpha}+\left(-i \Gamma_{11}-\gamma_{1}^{(2)}\right) \psi_{1}^{\beta} \psi_{1}^{\alpha 2}+\left(-i \Gamma_{13}-\gamma_{13}\right) \psi_{3}^{\beta} \psi_{3}^{\alpha} \psi_{1}^{\alpha}+\sum_{\nu} \kappa_{\nu} \psi_{1}^{\beta} \psi_{2, \nu}^{\alpha} \\
\left(-\mathcal{K}_{1}-\frac{\gamma_{1}^{(1)}}{2}\right) \psi_{1}^{\beta}+\left(i \Gamma_{11}-\gamma_{1}^{(2)}\right) \psi_{1}^{\alpha} \psi_{1}^{\beta 2}+\left(i \Gamma_{13}-\gamma_{13}\right) \psi_{3}^{\alpha} \psi_{3}^{\beta} \psi_{1}^{\beta}+\sum_{\nu} \kappa_{\nu}^{*} \psi_{1}^{\alpha} \psi_{2, \nu}^{\beta} \\
\vdots \\
\left(\mathcal{K}_{2, \nu}-\frac{\gamma_{2, \nu}^{(1)}}{2}-i \Gamma_{1 ; 2, \nu} \psi_{1}^{\beta} \psi_{1}^{\alpha}-i \Gamma_{3 ; 2, \nu} \psi_{3}^{\beta} \psi_{3}^{\alpha}\right) \psi_{2, \nu}^{\alpha}-\frac{\kappa_{\nu}^{*}}{2} \psi_{1}^{\alpha 2}+\Omega_{\nu} \psi_{3}^{\alpha} \\
\left(-\mathcal{K}_{2, \nu}-\frac{\gamma_{2, \nu}^{(1)}}{2}+i \Gamma_{1 ; 2, \nu} \psi_{1}^{\beta} \psi_{1}^{\alpha}+i \Gamma_{3 ; 2, \nu} \psi_{3}^{\beta} \psi_{3}^{\alpha}\right) \psi_{2, \nu}^{\beta}-\frac{\kappa_{\nu}}{2} \psi_{1}^{\beta 2}+\Omega_{\nu}^{*} \psi_{3}^{\beta} \\
\vdots \\
\left(\mathcal{K}_{3}-\frac{\gamma_{3}^{(1)}}{2}\right) \psi_{3}^{\alpha}+\left(-i \Gamma_{33}-\gamma_{3}^{(2)}\right) \psi_{3}^{\beta} \psi_{3}^{\alpha 2}+\left(-i \Gamma_{13}-\gamma_{13}\right) \psi_{1}^{\beta} \psi_{1}^{\alpha} \psi_{3}^{\alpha}-\sum_{\nu} \Omega_{\nu}^{*} \psi_{2, \nu}^{\alpha} \\
\left(-\mathcal{K}_{3}-\frac{\gamma_{3}^{(1)}}{2}\right) \psi_{3}^{\beta}+\left(i \Gamma_{33}-\gamma_{3}^{(2)}\right) \psi_{3}^{\alpha} \psi_{3}^{\beta 2}+\left(i \Gamma_{13}-\gamma_{13}\right) \psi_{1}^{\beta} \psi_{1}^{\alpha} \psi_{3}^{\beta}-\sum_{\nu} \Omega_{\nu}^{*} \psi_{2, \nu}^{\beta}
\end{array}\right)
$$

where $\mathcal{K}_{j}=-i / \hbar\left(\hat{T}_{j}+\hat{V}_{j}\right)$, and $\Gamma_{i j}=U_{i j} / \hbar$. The terms proportional to the density of the excited molecular states have been dropped, as these upper states are going to be adiabatically eliminated. The diffusion matrix $D$ is given by:

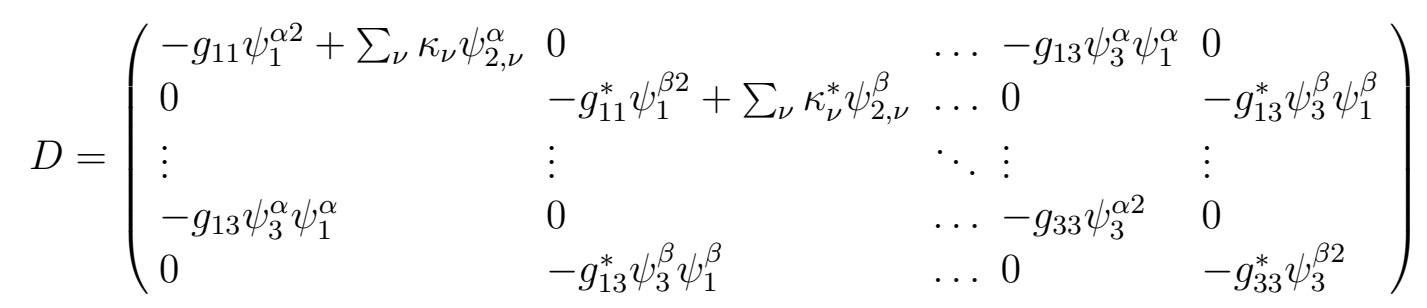

where $g_{i j}=\gamma_{i j}^{(2)}+i \Gamma_{i j}$ combines the atomic interactions and the two-body losses. The noise terms due to the self interaction of the excited molecular states have been ignored, as the density of these states is about to be assumed to be extremely small. This will enable them to be adiabatically eliminated. 
The Fokker-Planck equation leads to the following set of equations for the excited molecular states:

$$
\begin{aligned}
& \frac{\partial \psi_{2, \nu}^{\alpha}}{\partial t}=\left(\mathcal{K}_{2, \nu}-\frac{\gamma_{2, \nu}^{(1)}}{2}-i \Gamma_{1 ; 2, \nu} \psi_{1}^{\beta} \psi_{1}^{\alpha}-i \Gamma_{3 ; 2, \nu} \psi_{3}^{\beta} \psi_{3}^{\alpha}\right) \psi_{2, \nu}^{\alpha}-\frac{\kappa_{\nu}^{*}}{2} \psi_{1}^{\alpha 2}+\Omega_{\nu} \psi_{3}^{\alpha} \\
& \frac{\partial \psi_{2, \nu}^{\beta}}{\partial t}=\left(-\mathcal{K}_{2, \nu}-\frac{\gamma_{2, \nu}^{(1)}}{2}+i \Gamma_{1 ; 2, \nu} \psi_{1}^{\beta} \psi_{1}^{\alpha}+i \Gamma_{3 ; 2, \nu} \psi_{3}^{\beta} \psi_{3}^{\alpha}\right) \psi_{2, \nu}^{\beta}-\frac{\kappa_{\nu}}{2} \psi_{1}^{\beta 2}+\Omega_{\nu}^{*} \psi_{3}^{\beta}
\end{aligned}
$$

In order for two lasers to provide a two-photon transition without populating the excited MBEC, the single photon detunings $\delta_{\nu}$ must be made very large. This means that the population of the excited level is very small and that its time derivative can be ignored. If the terms $\partial_{t} \psi_{2, \nu}^{\alpha, \beta}$ and $\hat{T} \psi_{2, \nu}^{\alpha, \beta}$ in the equations of motion for $\psi_{2, \nu}^{\alpha, \beta}$ are ignored on the basis that they are smaller than the other terms, it becomes possible to write solutions for $\psi_{2, \nu}^{\alpha, \beta}(x, t)$ explicitly in terms of $\psi_{1}^{\alpha, \beta}(x, t)$ and $\psi_{3}^{\alpha, \beta}(x, t)$ :

$$
\begin{aligned}
& 0=\left(-\frac{i}{\hbar} V_{2, \nu}-\gamma_{2, \nu}^{(1)} / 2-i \Gamma_{1 ; 2, \nu} \psi_{1}^{\beta} \psi_{1}^{\alpha}-i \Gamma_{3 ; 2, \nu} \psi_{3}^{\beta} \psi_{3}^{\alpha}\right) \psi_{2, \nu}^{\alpha}-\frac{\kappa_{\nu}^{*}}{2} \psi_{1}^{\alpha 2}+\Omega_{\nu} \psi_{3}^{\alpha} \\
& 0=\left(\frac{i}{\hbar} V_{2, \nu}-\gamma_{2, \nu}^{(1)} / 2+i \Gamma_{1 ; 2, \nu} \psi_{1}^{\beta} \psi_{1}^{\alpha}+i \Gamma_{3 ; 2, \nu} \psi_{3}^{\beta} \psi_{3}^{\alpha}\right) \psi_{2, \nu}^{\beta}-\frac{\kappa_{\nu}}{2} \psi_{1}^{\beta 2}+\Omega_{\nu}^{*} \psi_{3}^{\beta} .
\end{aligned}
$$

A rearrangement of these equations, and the further assumption that the single-photon detuning $\delta_{\nu}$ is larger than the interaction terms $\Gamma_{i ; 2, \nu} \psi_{i}^{\beta} \psi_{i}^{\alpha}$, the excited state loss rate $\gamma_{2, \nu}^{(1)}$ and the trapping potential (i.e. $V_{2, \nu} \approx \hbar \delta_{\nu}$ ), gives the following result:

$$
\begin{aligned}
\psi_{2, \nu}^{\alpha} & =\frac{i / 2 \kappa_{\nu} \psi_{1}^{\alpha}{ }^{2}-i \Omega_{\nu} \psi_{3}^{\alpha}}{\delta_{\nu}} \\
\psi_{2, \nu}^{\beta} & =\frac{-i / 2 \kappa_{\nu}^{*} \psi_{1}^{\beta 2}+i \Omega_{\nu}^{*} \psi_{3}^{\beta}}{\delta_{\nu}} .
\end{aligned}
$$

These equations can then be substituted into Eq.(6) and Eq.(7) to produce the drift and diffusion matrices for the two levels $|1\rangle$ and $|3\rangle$. It is then possible to find a matrix $B$ such that $D=B \cdot B^{T}$. In general this matrix will not be unique. Finally, there is a theorem which allows us to map the solution of the master equation in Eq.(四) to the following set of Itô stochastic field equations:

$$
\begin{aligned}
\frac{\partial \psi_{1}^{\alpha}}{\partial t}= & \left(\mathcal{K}_{1}-\frac{\gamma_{1}^{(1)}}{2}-\left(g_{1}-i \Gamma\right) \psi_{1}^{\beta} \psi_{1}^{\alpha}-g_{13} \psi_{3}^{\beta} \psi_{3}^{\alpha}\right) \psi_{1}^{\alpha}-i \chi \psi_{1}^{\beta} \psi_{3}^{\alpha} \\
& +\sqrt{-i \chi \psi_{3}^{\alpha}-\left(g_{1}-i \Gamma\right) \psi_{1}^{\alpha 2}} \eta_{1}-\sqrt{g_{13} \psi_{1}^{\alpha} \psi_{3}^{\alpha}} \zeta_{1}^{*} \\
\frac{\partial \psi_{1}^{\beta}}{\partial t}= & \left(-\mathcal{K}_{1}-\frac{\gamma_{1}^{(1)}}{2}-\left(g_{1}^{*}+i \Gamma\right) \psi_{1}^{\beta} \psi_{1}^{\alpha}-g_{13}^{*} \psi_{3}^{\beta} \psi_{3}^{\alpha}\right) \psi_{1}^{\beta}+i \chi^{*} \psi_{1}^{\alpha} \psi_{3}^{\beta} \\
& +\sqrt{i \chi^{*} \psi_{3}^{\beta}-\left(g_{1}^{*}+i \Gamma\right) \psi_{1}^{\beta 2}} \eta_{2}-\sqrt{g_{13} \psi_{1}^{\beta} \psi_{3}^{\beta}} \zeta_{2} \\
\frac{\partial \psi_{3}^{\alpha}}{\partial t}= & \left(\mathcal{K}_{3}-\frac{\gamma_{3}^{(1)}}{2}+i \lambda-g_{3} \psi_{3}^{\beta} \psi_{3}^{\alpha}-g_{13} \psi_{1}^{\beta} \psi_{1}^{\alpha}\right) \psi_{3}^{\alpha}-\frac{i}{2} \chi^{*} \psi_{1}^{\alpha 2}
\end{aligned}
$$




$$
\begin{aligned}
& +\sqrt{-g_{3}} \psi_{3}^{\alpha} \eta_{3}+\sqrt{g_{13} \psi_{1}^{\alpha} \psi_{3}^{\alpha}} \zeta_{1} \\
\frac{\partial \psi_{3}^{\beta}}{\partial t}= & \left(-\mathcal{K}_{3}-\frac{\gamma_{3}^{(1)}}{2}-i \lambda-g_{3}^{*} \psi_{3}^{\beta} \psi_{3}^{\alpha}-g_{13}^{*} \psi_{1}^{\beta} \psi_{1}^{\alpha}\right) \psi_{3}^{\beta}+\frac{i}{2} \chi \psi_{1}^{\beta 2} \\
& +\sqrt{-g_{3}^{*}} \psi_{3}^{\beta} \eta_{4}+\sqrt{g_{13}^{*} \psi_{1}^{\beta} \psi_{3}^{\beta}} \zeta_{2}^{*}
\end{aligned}
$$

where

$$
\begin{aligned}
& \Gamma=\sum_{\nu} \frac{\left|\kappa_{\nu}\right|^{2}}{2 \delta_{\nu}}, \\
& \chi=\sum_{\nu} \frac{\kappa_{\nu} \Omega_{\nu}}{\delta_{\nu}}, \\
& \lambda=\sum \nu \frac{\left|\Omega_{\nu}\right|^{2}}{\delta_{\nu}},
\end{aligned}
$$

and the $\eta_{\nu}$ are a set of real, Gaussian noise sources which are $\delta$-correlated in time and space:

$$
\overline{\eta_{i}(\mathbf{x}, t) \eta_{j}\left(\mathbf{x}^{\prime}, t^{\prime}\right)}=\delta_{i j} \delta\left(\mathbf{x}-\mathbf{x}^{\prime}\right) \delta\left(t-t^{\prime}\right),
$$

and $\zeta_{\nu}$ are a set of complex, Gaussian noise sources which are $\delta$-correlated in time and space:

$$
\overline{\zeta_{i}^{*}(\mathbf{x}, t) \zeta_{j}\left(\mathbf{x}^{\prime}, t^{\prime}\right)}=\delta_{i j} \delta\left(\mathbf{x}-\mathbf{x}^{\prime}\right) \delta\left(t-t^{\prime}\right)
$$

These stochastic equations allow us to generate any multitime time-normally ordered quantum field averages by averaging selected moments of these fields over a sufficiently large sample of trajectories [20].

$$
\begin{aligned}
& \left\langle\overleftarrow{T} \hat{\psi}^{\dagger}(x, t) \ldots \hat{\psi}^{\dagger}\left(x^{\prime}, t^{\prime}\right) \vec{T} \hat{\psi}^{\dagger}\left(x^{\prime \prime}, t^{\prime \prime}\right) \ldots \hat{\psi}^{\dagger}\left(x^{\prime \prime \prime}, t^{\prime \prime \prime}\right)\right\rangle \\
& \quad=\frac{\psi^{\beta}(x, t) \ldots \psi_{\beta}\left(x^{\prime}, t^{\prime}\right) \psi^{\alpha}\left(x^{\prime \prime}, t^{\prime \prime}\right) \ldots \psi_{\alpha}\left(x^{\prime \prime \prime}, t^{\prime \prime \prime}\right)}{}
\end{aligned}
$$

where $(\overleftarrow{T}) \vec{T}$ is the (anti-)chronological time ordering operator.

The Itô stochastic equations reduce to the GPE for this system if the noise terms are ignored. As can be seen later, this is not the case when using a different stochastic calculus, such as Stratonovich calculus, which reinterprets the noise terms and therefore requires corrections to the deterministic part of the equations. This reinforces the fact that the noise terms cannot be treated separately from the deterministic terms, as the same physics produces both of them. It is also worth reiterating that there is no useful interpretation of the individual trajectories of the simulation, only the ensemble averages.

Our equations of motion are very similar to those obtainable by a direct coupling between the BEC and the stable MBEC. The difference is the nonlinear light shift of the BEC, which is proportional to $\Gamma$, and the linear light shift of the MBEC, which is equal to $\lambda$.

\section{SEMICLASSICAL SUPERCHEMISTRY}

Examination of the semiclassical equations of motion obtained by dropping the noise terms from Eq.(12) shows that the behaviour is sensitive to the relative strengths of the 
non-linearities. In the limit that there are no third order nonlinearities it can be seen that if the population starts in the atomic field then there is a complete, one-way conversion to molecules. In the presence of atomic and molecular interactions, the third order nonlinearities dephase this evolution, and cause a revival of the atomic population. This behaviour is shown in Fig. 2, where the evolution is shown for different values of the atomic-molecular coupling rate.
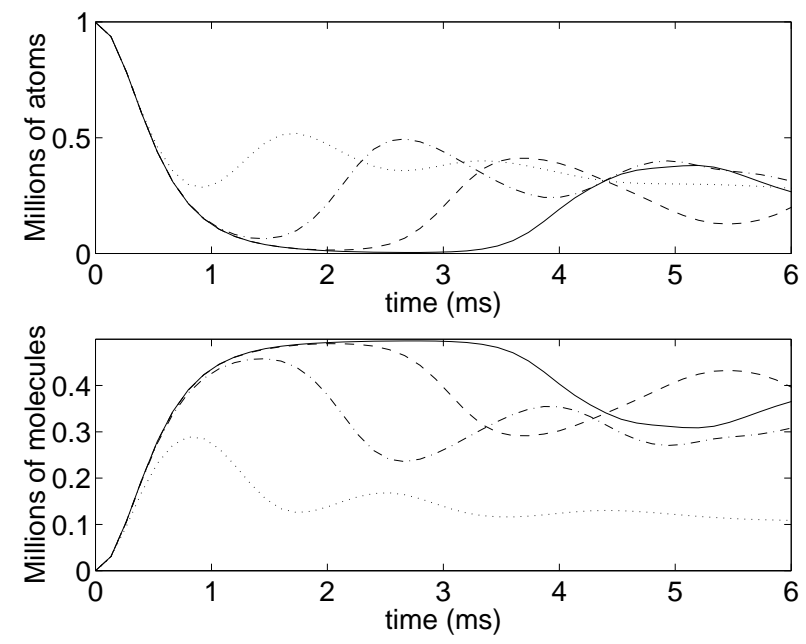

FIG. 2. Evolution of the semiclassical equations of motion for the atomic and molecular populations in a fixed three-dimensional harmonic trap. The values of $\chi$ used were $3.0 \times 10^{-5} \mathrm{~m}^{1 / 2} / \mathrm{s}$ (solid), $3.0 \times 10^{-6} \mathrm{~m}^{1 / 2} / \mathrm{s}$ (dashed), $3.0 \times 10^{-7} \mathrm{~m}^{1 / 2} / \mathrm{s}$ (dash-dotted) and $3.0 \times 10^{-8} \mathrm{~m}^{1 / 2} / \mathrm{s}$ (dotted).

This semiclassical behaviour has been observed in traveling-wave second harmonic generation, which is a zero-dimensional analogue of coupled atomic and molecular condensates. However, it has also been shown that the semiclassical approximation gives incorrect predictions for the mean behaviour of the fields in traveling-wave second harmonic generation [18, as well as for their quantum statistical properties [24,25]. This discrepancy is most pronounced when there is nearly complete conversion to the second harmonic, which occurs when the third order nonlinearities are very small [26]. It has since been shown that it is also possible to observe the effects of the quantum nature of the fields in a one-dimensional model of coupled atomic and molecular condensates [14].

The breakdown of the semiclassical approximation has a simple explanation. The semiclassical equations do not include the spontaneous emission terms which allow a molecule to break into two atoms in the absence of an atomic field. To include these processes in the model the full stochastic equations of motion must be considered, which include all the physics of the quantum fields. The noise terms of the evolution mimic the dephasing effect of third-order nonlinearities, and cause a revival of the atomic population.

For coupled atomic and molecular condensates in three dimensions, with losses due to background gas collisions and two-body collisions, it may be significantly more difficult to observe the quantum nature of the fields. In higher dimensions there is a larger volume of low density fields than in low dimensions, which in combination with the third order nonlinearities and the losses will tend to "blur out" the strong conversion to molecules which can be observed in the semiclassical theory for low dimensions. This effect can be counteracted by ensuring that the field is as dilute as possible, which makes the (second 
order in the fields) coupling between the BEC and the MBEC relatively stronger than the (third order in the fields) interaction terms. In order to minimise the diffusion due to kinetic energy and further reduce the effect of the interactions, the Raman transition must be made as strong as possible.

Making the Raman transition as strong as possible requires maximum laser intensities and a two-photon resonant coupling $(\Delta=\lambda)$. The practical limits on the total strength of the coupling are the requirement that the excited molecular state must not have significant population, and the need to avoid inducing large, attractive atomic interactions through the non-linear light shift due to the laser-assisted photoionisation. Unless there is a low excited state population, spontaneous losses from that highly energetic state could disrupt the condensates, and the adiabatic approximation used to eliminate the upper level may break down. This limit imposes the condition that the single photon detuning $\delta$ must be significantly larger than the Rabi frequencies of the individual laser beams. The second requirement means that the non-linear light shift should stay of the order of the repulsive interactions. If the Rabi frequency of the first laser is chosen such that $\Gamma=\Gamma_{11}$, then the nonlinear light shift will completely cancel the interactions between the atoms. There will still be repulsive interactions between the molecules and attraction between the atoms and the molecules.

Dilute condensates can be created by evaporating below the BEC condition using strong straps, and then adiabatically lowering the trap strengths in all directions. The trap depth remains large compared to the chemical potential of the condensate even for extremely weak traps [27]. The field cannot be arbitrarily dilute, however, as the accurate measurement of the fields depends on a sufficient column density. Many current experiments use highly anisotropic "cigar-shaped" traps, which allow a high column density along the long axis. For a given aspect ratio $\omega_{x}=\omega_{y}=A \omega_{z}$ and number of trapped atoms $N$, it is possible to calculate the transverse trap frequency required to obtain the required peak column density $\sigma_{p}:$

$$
\omega_{x}=\left(\frac{18 \sigma_{p}^{5} U_{11}^{2} \pi^{3}}{125 N^{3} m^{2} A^{2}}\right)^{\frac{1}{4}} .
$$

The peak density scales inversely with the aspect ratio $A$, which has been as large as 100 in current experiments [28]. In the next section the behaviour of the quantum model of the system will be investigated in both dilute and strong traps, and with both weak and strong coupling.

\section{QUANTUM SUPERCHEMISTRY}

This section will examine the difference between the quantum solution and the semiclassical solution allowing for physical constraints such as limited detuning and laser power. To ensure that the adiabatic approximation is being made self-consistently, the density of atoms in the excited state (which must remain small) can be calculated from the amplitudes of the other fields.

Since our last calculation [14], the interaction between the atomic and molecular species has been measured by examining the resonances in the molecular formation due to photoionisation [5]. The atoms are attracted to each other with a scattering length of 
$a_{13}=-180 \pm 150 a_{o}$, which leads to a value of $U_{13}=6 \pi a_{13} \hbar^{2} / m=-1.4 \times 10^{-50} \mathrm{~J} \mathrm{~m}^{3}$. The strength of the interactions between the $\mathrm{Rb}_{2}$ molecules has not been measured, so it will be assumed that they are the same as the interatomic interactions for the purposes of this calculation $\left(U_{11}=U_{33}=3 \times 10^{-51} \mathrm{~J} \mathrm{~m}^{3}\right)$.

The losses due to background gases are very slow on the timescale of the superchemistry. All calculations in this work use a value of $\gamma_{1}^{(1)}=\gamma_{3}^{(1)}=0.01 s^{-1}$, which is a comfortable overestimate of the background gas losses measured by the group at JILA [29]. At the high densities involved in the production of an atomic BEC, the three-body losses are dominant and the two-body losses are negligible $\left(\gamma_{1}^{(2)} \leq 1.6 \times 10^{-22} \mathrm{~m}^{3} / \mathrm{s}\right)$. By contrast, the threebody losses can be ignored at the low densities considered in this work, but in the presence of molecules the two-body losses cannot be ignored, as there is a significant cross-section for the inelastic scattering of an atom from a molecule. This was recently measured to be $\gamma_{13}^{(2)}<8 \times 10^{-17} \mathrm{~m}^{3} / \mathrm{s}$, and in this paper the upper bound shall be used in all calculations.

It is experimentally difficult to produce large Rabi frequencies for the atom-molecule interaction due to the low Franck-Condon factors, but this does not appear to be a limitation of this system provided large Rabi frequencies can be achieved for the molecule-molecule transition.

\section{A. Numerical methods}

Although Itô equations of motion for this system have been produced in the previous section, it is much easier to use high-order integration methods on Stratonovich equations. This is because the normal chain rule of differentiation applies to Stratonovich calculus, and standard high-order methods can be used without modification. The transformation from a set of Itô equations to a set of Stratonovich equations can be performed easily, as it involves a simple correction to the deterministic terms. Unfortunately, this correction is infinite for Eq.(12). This is a recurrence of the renormalisation problem often found in quantum field theories.

The infinite correction only occurs in the continuous field description. When the conversion to Stratonovich calculus is done after the fields have been discretised and placed on a rectangular grid with spacing of $\Delta x, \Delta y$ and $\Delta z$ in the three dimensions, the following correction terms are obtained:

$$
\left.\left(\begin{array}{c}
\frac{\partial \psi_{1}^{\alpha}}{\partial t} \\
\frac{\partial \psi_{1}^{\beta}}{\partial t} \\
\frac{\partial \psi_{3}^{\alpha}}{\partial t^{\beta}} \\
\frac{\partial \psi_{3}^{\beta}}{\partial t}
\end{array}\right)\right|_{\text {correction }}=\left(\begin{array}{c}
\frac{g_{13} / 2+g_{1}}{2 \Delta x \Delta y \Delta z} \psi_{1}^{\alpha} \\
\frac{g_{13}^{*} / 2+g_{1}^{*}}{2 \Delta x \Delta y z} \psi_{1}^{\beta} \\
\frac{g_{13} / 2+g_{3}}{2 \Delta x \Delta y \Delta} \psi_{3}^{\alpha} \\
\frac{g_{13}^{*} / 2+g_{3}^{*}}{2 \Delta x \Delta y \Delta z} \psi_{3}^{\beta}
\end{array}\right) .
$$

This correction is equivalent to a stepsize-dependent phase shift of the fields. It is clear that the correction is infinite in the limit of zero spatial cell size. In fact, this limit is not realistic for atomic fields, as the interactions between the particles are modeled as a contact potential, and this approximation fails at a sufficiently small distance scale. This puts a lower limit on the distance between the gridpoints, which will be of the order of magnitude of the scattering length. This forced discretisation is equivalent to the cutoff in momentum which is usually used to solve renormalisation problems. 
In a numerical simulation with stepsize $\Delta t$, the noise terms $\eta(\mathbf{x}, t)$ are included at each timestep by choosing a random number $R$ from a Gaussian distribution centred around zero and with unit width. The noise terms are then formed using $\eta(x, t)=R / \sqrt{\Delta x \Delta y \Delta z \Delta t}$. This means that going to smaller grid spacing slows the computation in three ways. The field contains more points, which proportionally increases the computation time per timestep. Also, the noise terms become larger, which means that both a smaller timestep is required to perform a stable integration and a larger ensemble of paths is required to obtain precise averages. It follows that these stochastic methods are most successful when the density is low. Fortunately, the strong effect should also be in this limit.

The stochastic integration was performed with the XMDS package, developed by Collecutt and Drummond [30]. In collaboration with them and the ANU Supercomputer Facility, this has been adapted to run multiple integrations of the stochastic equations in parallel on the APAC supercomputer [31].

\section{B. Quantum superchemistry with varying coupling rates}

The feasibility of observing quantum field effects shall be demonstrated by considering the most dilute system possible. The diluteness will be limited by the requirement that the column density be large enough to provide easy measurements, and by the total number of atoms available in the initial trap. All further calculations assume that it will be possible to have a million atoms in the trap after the adiabatic expansion. The noise in the column density measurements is estimated to be $10^{13} \mathrm{~m}^{-2}$, which is that of the current BEC at ANU [32]. For the purposes of this subsection, it is assumed that the trap has an aspect ratio of $A=30$. The weakest trap in which $99.5 \%$ of the atoms are above the noise level in the column density has a trap frequency of $\omega_{x}=0.59 \mathrm{~s}^{-1}$, and a condensate size of $0.09 \mathrm{~mm}$ in the strong axis and $0.3 \mathrm{~mm}$ in the weak direction. The effect of varying the coupling rate in such a trap will be calculated in this subsection. The next subsection will examine the quantum field effects in stronger traps.

Fig.(3) compares the evolution of the atomic population with the solution to the GrossPitaevskii equation. It can be seen that the effect of the quantum statistics of the field grows

as the coupling increases. The coupling cannot be increased indefinitely, due to restrictions on both the available laser power and allowable detuning. 


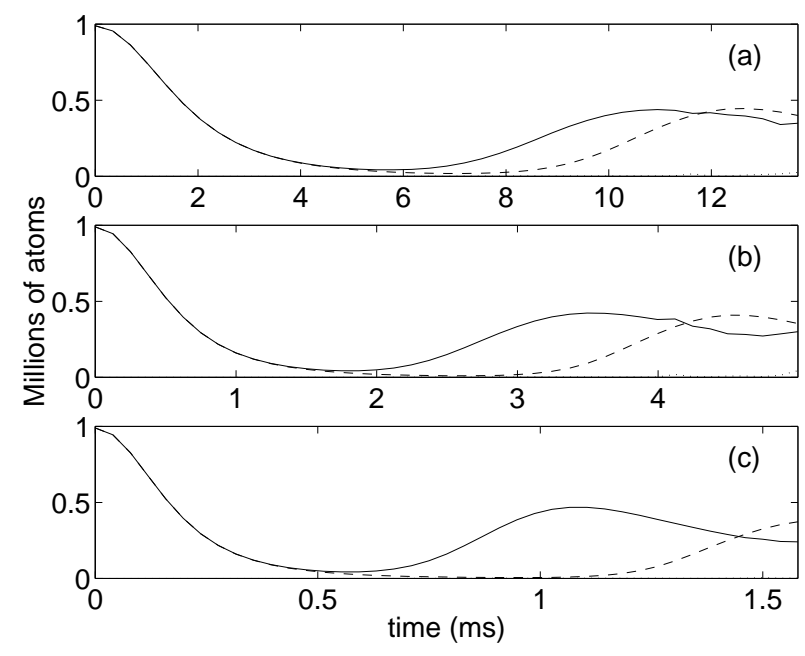

FIG. 3. Evolution of the atomic population (solid lines) compared to the solution of the GPE (dashed lines). Trap parameters are as given in the text. In each case the atomic-molecular coupling $\kappa$ was chosen such that $\Gamma=\Gamma_{11}$, and the detuning $\delta$ was chosen to be 50 times larger than the molecule-molecule Rabi frequencies $\Omega$. Thus, a larger coupling strength requires a higher detuning. (a) $\chi=4.7 \times 10^{-6} \mathrm{~m}^{3 / 2} \mathrm{~s}^{-1}$, which corresponds to a detuning of $160 \mathrm{MHz}$, (b) $\chi=1.5 \times 10^{-5} \mathrm{~m}^{3 / 2}$ $\mathrm{s}^{-1}$, which corresponds to a detuning of $1.6 \mathrm{GHz}$, (c) $\chi=4.7 \times 10^{-5} \mathrm{~m}^{3 / 2} \mathrm{~s}^{-1}$, which corresponds to a detuning of $16 \mathrm{GHz}$. The dotted lines, where visible, are the uncertainties in the theory due to the sampling error.

The difference between the dynamics of the system and the semiclassical result is essentially unaffected by the losses, which dominate at higher densities and over longer timescales. By contrast, the inclusion of the atom-molecule interactions in this model has reduced the distinction at high densities. From Fig.(3) it can be seen that the deviation from the semiclassical result is clearly observable for currently achievable trap and coupling parameters.

\section{Quantum superchemistry at varying densities}

Although it is possible to use very weak traps to work in the low density limit, most experiments are performed using stronger traps than those modeled in the previous section. This subsection will examine the effects of changing the density.

The most dramatic effect of increasing the strength of the trap in the model is that the calculation becomes less stable. As previously explained, the noise in the numerical simulation becomes relatively larger as the unit cell size decreases. Stronger traps mean that the overall size of the condensate decreases, and so does the minimum allowable stepsize in the spatial grid. It therefore becomes harder to use the stochastic field equations to model the system for long times. The stochastic method will always produce results up to a certain point in time, but as the individual trajectories become unstable numerically it becomes impossible to integrate further.

Under the constraint that there are just enough atoms to provide easily measurable results, the ratio of the second-order and third-order nonlinearities scales as $\omega_{x}^{2 / 3}$, whereas the condensate volume will scale as $\omega_{x}^{-2}$. The difference between the full, quantum evolution 
of the system and the semiclassical evolution depends on the ratio of the second-order and third-order nonlinearities, so based on this scaling the distinction may be measurable for trap strengths where the positive-P method cannot calculate the correct result.

Fig. (4) shows the evolution of the atomic population for three different trap frequencies. The aspect ratio of the trap and the coupling rate are kept the same. The number of atoms in the trap is varied so that each trap contains the minimum number of atoms required to maintain $99.5 \%$ of them above a column density of $\sigma=10^{13} \mathrm{~m}^{-2}$. An approximate method of calculating this is that the number of atoms $N$ required to obtain a peak density of $\sigma_{p}$ is

$$
N=\left(\frac{18 U_{11}^{2} \sigma_{p}^{5}}{125 \omega_{x}^{4} M^{2} A^{2}}\right)^{1 / 3}
$$

The figure shows a significant effect of the quantum statistics of the field even for much higher densities. Unfortunately, it also shows the difficulty in performing quantitative experiments at these densities, where the theoretical results are difficult or impossible to obtain by the stochastic methods used in this paper. In each plot, the integration cannot be performed far beyond the region shown.

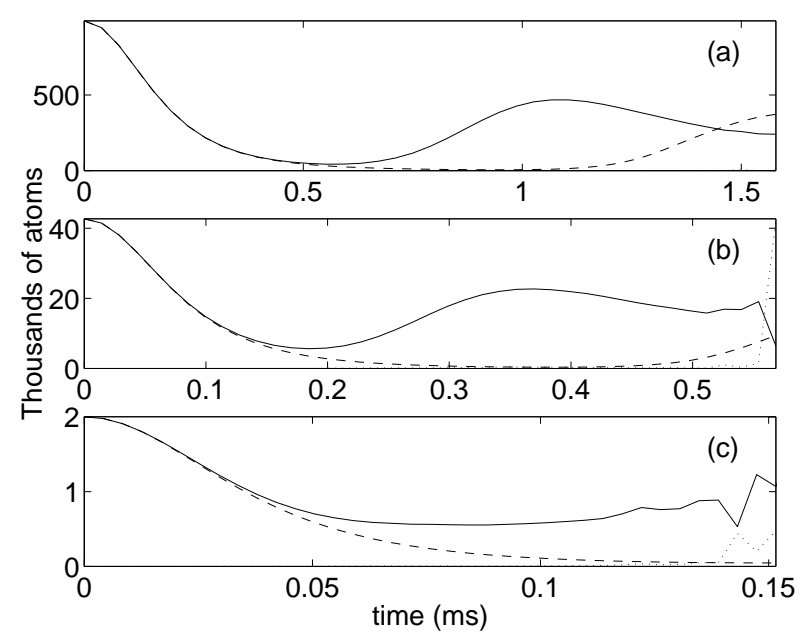

FIG. 4. Evolution of the atomic population (solid lines) compared to the solution of the GPE (dashed lines). The parameters used are $\Gamma=\Gamma_{11}, \chi=4.7 \times 10^{-5} \mathrm{~m}^{3 / 2} \mathrm{~s}^{-1}$, which corresponds to a detuning of $16 \mathrm{GHz}$. The trap has an aspect ratio of $A=30$, and trap frequencies (a) $\omega_{x}=2 \pi \times 0.092 \mathrm{~Hz}$, (b) $\omega_{x}=2 \pi \times 1.0 \mathrm{~Hz}$, and (c) $\omega_{x}=2 \pi \times 10 \mathrm{~Hz}$. The dotted lines, where visible, are the uncertainties in the theory due to the sampling error.

The importance of including the quantum statistical effects actually appears to increase with density. This is surprising, as we would expect the third-order nonlinearities to be the main source of dephasing of the conversion, and they are getting stronger with density. This may be due to the fact that the densities considered here are so low that the main dephasing is being caused by the kinetic energy term. If this is true, then there will be an optimal density which will maximise the parametric conversion rate compared to the kinetic energy terms, but minimise the two-body interaction terms with respect to the conversion rate. Detailed testing of this hypothesis is difficult due to the numerical difficulties, and is beyond the scope of this work. 


\section{CONCLUSIONS}

This paper has shown that it is possible to observe the quantum statistical effects of the field in coupled atomic and molecular BEC. The effect is most clearly visible in the low-density and high-coupling strength limits.

Although it is a very successful model, the GPE cannot be applied to every system of coupled BECs. While it may seem reasonable to expect that the quantum statistics will tend to affect the multi-time correlations of the field rather than the mean field, our result shows it is sometimes also important to include them when considering the equations of motion for moments of the mean field. The signature of the breakdown of the GPE occurs in the simplest experimental observable - the total atomic and molecular populations.

The calculations in this paper were numerically intensive. Although the positive-P representation contains the full description of the quantum field, it cannot be applied to every system of coupled BECs. Individually, trajectories do not have to behave in a physical fashion and they can become unstable over time in the absence of damping. The technique is therefore often only useful over short timescales, or for examining systems which are well described by the coherent state basis which underlies the description. This is not usually a natural basis of the atomic field, although it is often a good basis for the optical field. A possible counterexample may be a continuously pumped atom laser operating well above threshold, for which the positive-P representation may be tractable. It is also to be expected that the behaviour of such a device would depend critically on the quantum statistics.

\section{ACKNOWLEDGMENTS}

This research was supported by the Australian Research Council. I would like to thank Greg Collecutt and Peter Drummond for their useful numerical integration package XMDS, which I recommend. I would like to thank Greg again, as well as Margaret Kahn and David Singleton from the ANU Supercomputer Facility for their help in adapting it to work on

a parallel computer. I would also like to thank Craig Savage, John Close, Jessica Lye and Cameron Fletcher for their informative discussions. 


\section{REFERENCES}

[1] M.H. Anderson et al., Science 269, 198 (1995).

[2] B. Levi, Physics Today 53 No. 9, 46 (2000).

[3] P.D.Drummond, K.V. Kheruntsyan and H. He, Phys. Rev. Lett. 81, 3055 (1998).

[4] D.J. Heinzen, R. Wynar, P.D. Drummond and K.V. Kheruntsyan, Phys. Rev. Lett. 84, 5029, (2000).

[5] R. Wynar, R.S. Freeland, D.J. Han, C. Ryu, D.J. Heinzen, Science 287, 1016 (2000).

[6] P.S. Julienne, K. Burnett, Y. B. Band and W. C. Stwalley, Phys. Rev. A 58, R797 (1998).

[7] J. Javanainen and M. Mackie, Phys. Rev. A 58, R789 (1998).

[8] J. Javanainen and M. Mackie, Phys. Rev. A 59, R3186 (1999).

[9] M. Mackie, R. Kowalski and J. Javanainen, Phys. Rev. Lett. 84, 3803, (2000).

[10] J.J. Hope, M.K. Olsen and L.I. Plimak, Phys. Rev. A 63,043603(2001).

[11] M. Holland, J. Park and R. Walser, Phys. Rev. Lett. 86,1915(2001).

[12] M.K. Olsen, J.J. Hope and L.I. Plimak, Phys. Rev. A 64,013601(2001).

[13] K. Góral, M. Gajda and K. Rzạzewski, Phys. Rev. Lett. 86,1397(2001).

[14] J.J. Hope and M.K. Olsen, Phys. Rev. Lett. 86,3220(2001).

[15] L.P. Pitaevskii, Zh. Eksp. Teor. Fiz. 40, 646 (1961). E.P. Gross, Nuovo Cimento 20, 454 (1961).

[16] R.J. Ballagh, K. Burnett and T.F. Scott, Phys. Rev. Lett. 78, 1607 (1997).

[17] D. Walls and G. Milburn, Quantum Optics, Springer-Verlag, Berlin, 1994.

[18] M.K. Olsen, R.J. Horowicz, L.I. Plimak, N. Treps and C. Fabre, Phys. Rev. A 61, 021803(R) (2000).

[19] P.D. Drummond and C.W. Gardiner, J. Phys. A 13, 2353 (1980).

[20] M.J. Steel et al, Phys. Rev. A 58, 4824, (1998).

[21] P.D. Drummond and J.F. Corney, Phys. Rev. A 60, R2661 (1999).

[22] U.V. Poulsen and K. Molmer, Phys. Rev. A 63,023604(2001).

[23] R. Graham and H. Haken, Z. Phys. 234, 193 (1970); 235166 (1970).

[24] M.K. Olsen and R.J. Horowicz, Opt. Commun. 168, 135, (1999).

[25] M.K. Olsen, L.I. Plimak, M.J. Collett and D.F. Walls, Phys. Rev. A 62, 023802 (2000).

[26] M.K. Olsen, V.I. Kruglov and M.J. Collett, unpublished.

[27] C. Fletcher, private communication.

[28] A. Görlitz et al., cond-mat/0104549; R. Onofrio et al., Phys. Rev. Lett. 84, 810 (2000).

[29] E.A. Burt et al., Phys. Rev. Lett. 79, 337 (1997).

[30] G.R. Collecutt, P.D. Drummond and H. He, http://www.physics.uq.edu.au/xmds/

[31] http://nf.apac.edu.au/

[32] J. Lye, private communication. 